\title{
Correction to: Connectivity map-based drug repositioning of bortezomib to reverse the metastatic effect of GALNT14 in lung cancer
}

Ok-Seon Kwon · Haeseung Lee $(D) \cdot$ Hyeon-Joon Kong • Eun-Ji Kwon • Ji Eun Park • Wooin Lee (D) Seungmin Kang • Mirang Kim (D) - Wankyu Kim • Hyuk-Jin Cha (D)

Published online: 11 February 2021

(c) The Author(s), under exclusive licence to Springer Nature Limited 2021

Correction to: Oncogene (2020) 39:4567-4580

https://doi.org/10.1038/s41388-020-1316-2

In the originally published version, the grant number of Dr Kim Wankyu was wrongly described in the original paper by mistake. This work was supported by a grant from the National Research Foundation of Korea (NRF-2017M3C9A5028691 from H-JC, NRF-2019R1C1C1008710 from O-SK and NRF2017M3C9A5028690 from WK).

The original version was corrected. 